\title{
Study on the Industrial Process of Rubber Anti-oxidant RD
}

\author{
Liu Yu ${ }^{\dagger, \hbar, *}$, Gao qinyu $u^{\dagger}$, Liu Lianxin ${ }^{\ddagger}$, and Shi guangxia \\ ${ }^{\dagger}$ College of Chemistry and Engineering Technology, China University of Mining and Technology, Xuzhou China 221116 \\ *E-mail: liuyu0138@163.com \\ †Xuzhou College of Industrial and Technology, Jiangsu Xuzhou China 221140
}

(Received June 30, 2011; Accepted August 25, 2011)

\begin{abstract}
This paper is on the industrial synthesis process of anti-oxidant RD ((2,2,4-trimethyl-1,2-dihydroquinoline polymer $\left(\mathrm{C}_{12} \mathrm{H}_{15} \mathrm{~N}\right)_{\mathrm{n}}$. $\left.\mathrm{n}=2-4\right)$ ). The content of dimer, trimer and tetramer of $\mathrm{RD}$ as the inspection targets, using the orthogonal design method - take the ratios of keto-amine, the reaction time, the reaction temperatures and the ratios of catalyst acid-amine as inspect factors - to optimized the reaction condition. The results indicate that the best ratio of keto-amine is $2: 1$, the time of salification and condensation is 3 hours and 7.5 hours. The range of temperature of salification and condensation is $135^{\circ} \mathrm{C}$ and $120-125^{\circ} \mathrm{C}$, and that the best ratio of acid-amine is 0.2 : 1 (the proportion is the concentration ratio for mole). Under the optimization conditions, the yield of RD was stabilized and content of $\mathrm{RD}$ more than $45 \%$.
\end{abstract}

Key words: Rubber anti-oxidant RD, Orthogonal design, Ratio of keto-amine, Ratio of catalyst acid-amine

\section{INTRODUCTION}

Rubber Anti-oxidant $\mathrm{RD}^{1,2}$ (2,2,4-trimethyl-1,2-dihydro-quinoline polymer, $\left(\mathrm{C}_{12} \mathrm{H}_{15} \mathrm{~N}\right) \mathrm{n}$. $\left.\mathrm{n}=2-4\right)$ is a ketone amine antioxidant. It has performs excellently on the protection of oxidation of rubber which is caused by thermal oxidative aging and catalytic oxidation by metal ions. RD is mainly used for natural rubber and chloroprene rubber etc. It has excellent effects to the ageing caused by heating and oxygen, and it has stronger restrained effect to the metallic catalysis and oxidation, it is Suitable for naturat, buna-s, buna-N and chloroprene rubbers. Rubber Antioxidant $\mathrm{RD}$ has a longer-time remaining of protective effect, because it has a higher molecular weight and has little diffusive loss. So it is suitable for the rubber products used in tropical area.

There is no influence on the processing properties of rubber, curing characteristics and physical properties of vulcanizates when adding rubber anti-oxidation RD. Anti-degradants for rubber comprising of a mixture consisting essentially of 2,2,4-trimethyl-1,2-dihydro-quinoline monomer, dimer thereof and more highly polymerized products than the dimer the contents of the quinoline dimer being less than $20 \mathrm{wt} \%$ and $30 \mathrm{wt} \%$ or even more, respectively. The anti-degradants for rubber are very useful for preventing both heat aging and flex cracking of rubber. There are two methods of synthesizing rubber Anti-oxidant RD at present. One is a "two-step process", 3 the equipment investment is too expensive for the long technical process in this method. The other method is a "one-step process"; the catalyst used in this process is expensive. Rubber Anti-oxidant RD polymer mixture is a so-called dihydro-quinoline polymer, produced by a reaction between aniline and acetone, which are widely used for heat aging resistance, since they are not only superior in preventing heat aging but also cheaply available. In this study, the "one-step process" with the catalyst of hydrochloric acid is used. The content of 2,2,4-trimethyl-1,2dihydro-quinoline polymer $\left(\left(\mathrm{C}_{12} \mathrm{H}_{15} \mathrm{~N}\right) \mathrm{n} . \mathrm{n}=2-4\right)$ is the inspection target, using the orthogonal design method, take the ratio of keto-amine, reaction time, reaction temperature, acid-amine ratio as inspects factors, product receives the rate is stable. Synthesis of antioxidant RD with superior quality was deterred.

\section{EXPERIMENTAL SECTION}

\section{Materials and Equipment}

Acetone and aniline are analytically pure. Hydrochloric acid (concentration 31\%-32\%), sodium hydroxide solution (concentration 32\%) HPLC-grade methanol was purchased from fisher. Hitachi L-2000 Hplc inclusion autosampler L-2200, organizer, uv-vis detector L-2420 (200 nm-900 nm), pump L-2130. Chromatographic column Shi$\operatorname{madzu} \mathrm{C} 184.6 \mathrm{~mm} \times 150 \mathrm{~mm} / 5 \mu \mathrm{m}$. Satorious electronic balance B204-N (Max $210 \mathrm{~g}$; d=0.1 mg). Ultrasound cleaner, 
<smiles>Nc1ccccc1</smiles><smiles>CC(=O)[I-]C(C)=O</smiles>

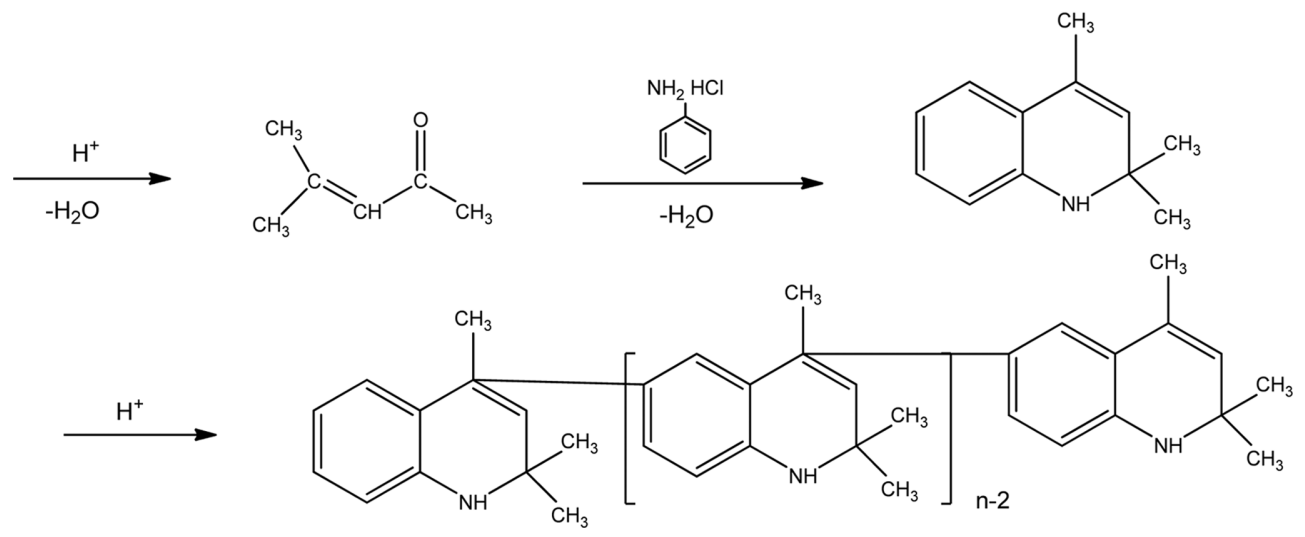

Fig. 1. principle of reaction.

Vacuum rotating evaporation apparatus etc.

\section{Principle of reaction and manufacture process}

The acetone and aniline reaction is shown in Fig. 1. It is well known that $\mathrm{RD}$ polymer is an excellent antioxidant for rubber, and particularly $\mathrm{RD}$ polymer containing a large amount of RD dimer has exhibited markedly superior performance. The product mainly contain dimer, trimer and tetramer of 2,2,4-trimethyl-1,2-dihydro-quinoline. The product quality depended on the content of dimer. The content of $\mathrm{RD}$ polymer is about $40 \mathrm{wt} \%$ to $50 \mathrm{wt} \%$. The manufacture process of Rubber Anti-oxidant RD has three stages, shown in Fig. 2.

(1) The reaction of aniline and acetone produces Rubber Anti-oxidant RD, hydrochloric acid as catalyst. The reactions are condensation and polymerization. The temperature of the reaction, the amount of acetone, aniline and catalyst should be controlled in the reaction. At the same time the adding method of acetone is very important others as well as little. In the first place hydrochloric acid reacted to aniline produced salt and the reaction time is about 3 hours. Removed the produced water in the reaction process. In the second, aniline or front fraction were added into the reactor under certain condition. Acetone of adding rate and method need control and adding time of about 5 to 6 hours.

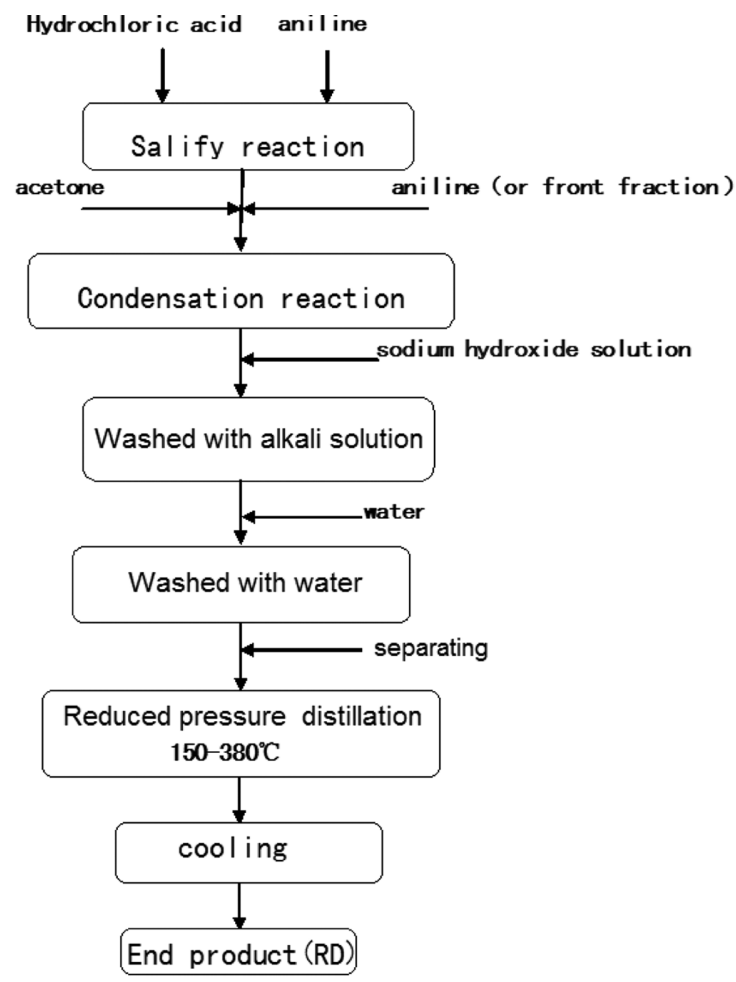

Fig. 2. Manufacture process of Rubber Anti-oxidant RD.

(2) In the reaction solution was added to the sodium hydroxide solution neutralize the excess hydrochloric 
acid, $\mathrm{pH}$ between 7 to 9 and washed with water. Volume of washing water is twice of reaction solution.

(3) The products are separated from unreacted material (aniline) and solvent(acetone) in the end. Unreacted material and solvent need purification and will be recycled to the reactor.

\section{Optimization of manufacture process}

The method for producing Rubber Anti-oxidant RD by means of the reaction of aniline and acetone under certain conditions. ${ }^{6-11}$

In the manufacture of polymerized 2,2,4-trimethyl-1,2dihydro-quinoline, reaction temperature, reaction temperature, the ratio of keto-amine, and the ratio of catalyst acid-amine are key factors. The polymer Content of 2,2,4trimethyl-1,2-dihydro-quinoline (dimer, trimer, tetramer) as investigate destination. Orthogonal experimental design determines the optimum reaction conditions. Level factors shown Table 1. A is the ratio of keto-amine, B is time of condensation reaction, $\mathrm{C}$ is temperature of condensation reaction and $\mathrm{D}$ is the ratio of catalyst of acid-amine.

According to the level and factors shown in Table 1 and Table 3, the choice of $\mathrm{L}_{9}\left(3^{4}\right)$ orthogonal test and take $9.3 \mathrm{~g}$ aniline, the other by adjusting the ratio in Table 3, to get the RD and dry. Test method of content of RD shown in below.

Test method of content of Rubber Anti-oxidant RD

Chemicals \& Reagent: Methanol HPLC Grade

Water HPLC Grade

Mobile phase: Methanol and water Filter and degas. Composition of mobile phase shown in Table 2.

Table 1. Factors and level of $\mathrm{L}_{9}\left(3^{4}\right)$

\begin{tabular}{ccccc}
\hline \multirow{2}{*}{ Level } & \multicolumn{4}{c}{ Factor } \\
\cline { 2 - 5 } & $\mathrm{A}(\mathrm{mole} / \mathrm{mole})$ & $\mathrm{B}($ time$/ \mathrm{h})$ & $\mathrm{C}\left({ }^{\circ} \mathrm{C}\right)$ & $\mathrm{D}($ mole $/$ mole $)$ \\
\hline 1 & 1.5 & 6.5 & $115-120$ & 0.15 \\
2 & 2.0 & 7.5 & $120-125$ & 0.20 \\
3 & 2.5 & 8.5 & $125-130$ & 0.25 \\
\hline
\end{tabular}

Table 2. Composition of mobile phase

\begin{tabular}{ccc}
\hline Time $\min$ & Methanol $\%$ & Water $\%$ \\
\hline 0 & 65 & 35 \\
10 & 65 & 35 \\
20 & 75 & 25 \\
30 & 85 & 15 \\
40 & 90 & 10 \\
50 & 95 & 5 \\
60 & 95 & 5 \\
70 & 100 & 0 \\
\hline
\end{tabular}

Table 3. Result from the orthogonal test of uniform design for manufacture process of $\mathrm{RD}$

\begin{tabular}{|c|c|c|c|c|c|}
\hline \multirow{2}{*}{ No. } & \multicolumn{4}{|c|}{ level } & \multirow{2}{*}{ content } \\
\hline & A & B & $\mathrm{C}$ & $\mathrm{D}$ & \\
\hline 1 & 1 & 1 & 1 & 1 & 33.35 \\
\hline 2 & 1 & 2 & 2 & 2 & 51.45 \\
\hline 3 & 1 & 3 & 3 & 3 & 44.25 \\
\hline 4 & 2 & 1 & 2 & 3 & 47.65 \\
\hline 5 & 2 & 2 & 3 & 1 & 46.30 \\
\hline 6 & 2 & 3 & 1 & 2 & 48.65 \\
\hline 7 & 3 & 1 & 3 & 2 & 32.55 \\
\hline 8 & 3 & 2 & 1 & 3 & 35.90 \\
\hline 9 & 3 & 3 & 2 & 1 & 46.75 \\
\hline$\overline{\mathrm{I}}_{j}$ & 43.017 & 37.850 & 39.300 & 42.133 & \\
\hline$\overline{\mathrm{II}}_{j}$ & 47.533 & 44.550 & 48.617 & 44.217 & \\
\hline$\overline{\mathrm{III}}_{j}$ & 38.400 & 46.550 & 41.033 & 42.600 & \\
\hline $\mathrm{R}$ & 9.133 & 8.700 & 9.317 & 2.084 & \\
\hline
\end{tabular}

\section{Preparation of test solution}

Weigh accurately about $1.5 \mathrm{mg}$ of Rubber Anti-oxidant $\mathrm{RD}$ in a $10 \mathrm{~mL}$ volumetric flask with methanol.

\section{Chromatographic Condition}

Instrument: HPLC equipped with Pump, Injector, UV detector and Recorder.

Column: C18 (4.6×250 mm), 5 um Wavelength: UV at $254 \mathrm{~nm}$.

Flow rate: $1.0 \mathrm{ml} / \mathrm{min}$. Injection volume: $20 \mu \mathrm{L}$. Run time: 70 minutes

Procedure: Inject $20 \mu \mathrm{L}$ of system suitability solution into the system and record the chromatograms up to 70 mins. Calculate the concentration of related substances by

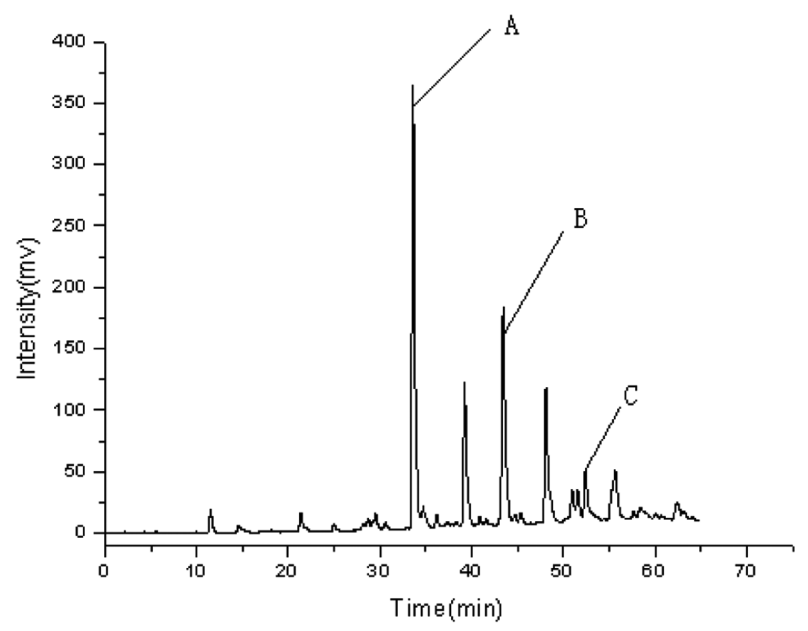

Fig. 3. HPLC chromatogram of Anti-oxidant RD. A: Dimer, B: Trimer, C: Tetramer 
Table 4. Tests of Between-Subjects Effects Dependent Variable: content of RD

\begin{tabular}{|c|c|c|c|c|c|}
\hline Source & Type III Sum of Squares & $\mathrm{df}$ & Mean Square & $\mathrm{F}$ & Sig. \\
\hline$\overline{\text { Corrected Model }}$ & 397.023(a) & 6 & 66.171 & 18.453 & 0.052 \\
\hline Intercept & 16628.102 & 1 & 16628.102 & 4637.165 & 0.000 \\
\hline Acetone ratio of aniline & 125.132 & 2 & 62.566 & 17.448 & 0.054 \\
\hline Time of reaction & 124.580 & 2 & 62.290 & 17.371 & 0.054 \\
\hline Temperature of reaction & 147.312 & 2 & 73.656 & 20.541 & 0.046 \\
\hline Error & 7.172 & 2 & 3.586 & & \\
\hline Total & 17032.298 & 9 & & & \\
\hline Corrected Total & 404.195 & 8 & & & \\
\hline
\end{tabular}

R Squared $=0.982$ (Adjusted R Squared $=0.929$ )

Table 5. Content of Rubber Anti-oxidant RD

\begin{tabular}{ccccc}
\hline No. & Dimer (\%) & Trimer (\%) & Tetramer (\%) & Total (\%) \\
\hline 1 & 26.264 & 17.534 & 4.320 & 48.118 \\
2 & 26.325 & 16.765 & 5.250 & 48.340 \\
3 & 27.427 & 15.925 & 5.465 & 48.817 \\
4 & 26.645 & 16.583 & 5.285 & 48.513 \\
5 & 26.895 & 16.662 & 5.185 & 48.742 \\
6 & 27.248 & 16.766 & 5.019 & 49.033 \\
\hline
\end{tabular}

area normalization method.

HPLC chromatogram of Anti-oxidant RD shown in Fig. 3.

\section{Result of experiment}

The experimental result shown in Table 3, content of dimer and trimer and tetramer. Orthogonal test showed that the ratio of the reaction temperature significantly affects the content, followed by the ratio keto-amine, reaction time and catalyst acid-amine. The result shows that $\mathrm{A}_{2} \mathrm{~B}_{3} \mathrm{C}_{2} \mathrm{D}_{2}$ has the significant effect on the reaction. But comparing the reaction time of $\mathrm{B}_{2}$ with $\mathrm{B}_{3}$, the content of RD only increased less than $2 \%$ as extending 1 hours. Economic considerations of energy that we are select $\mathrm{B}_{2}$. The optimal conditions of process for $\mathrm{A}_{2} \mathrm{~B}_{2} \mathrm{C}_{2} \mathrm{D}_{2}$. The ratio of keto-amine is 2.0 (mole/mole), the reaction time was 6 hours, the reaction temperature is $120-125^{\circ} \mathrm{C}$, the ratio of catalyst acid-amine 0.2 (mole/mole). Optimized conditions used in the production, testing 6 batches, the results are shown in Table 5. The content of Rubber Anti-oxidant $\mathrm{RD}$ is more than $45 \%(\mathrm{Wt} \%)$. The reaction has been improved content of dimer and trimer and tetramer while content of the dimer more than $26 \%$. Tests of between-subject's effects by spss 12.0 . The calculated results shown that in Table 4 .

\section{DISCUSSION}

The first place of industrial process aniline reacted with hydrochloric acid under certain conditions. ${ }^{12,13}$

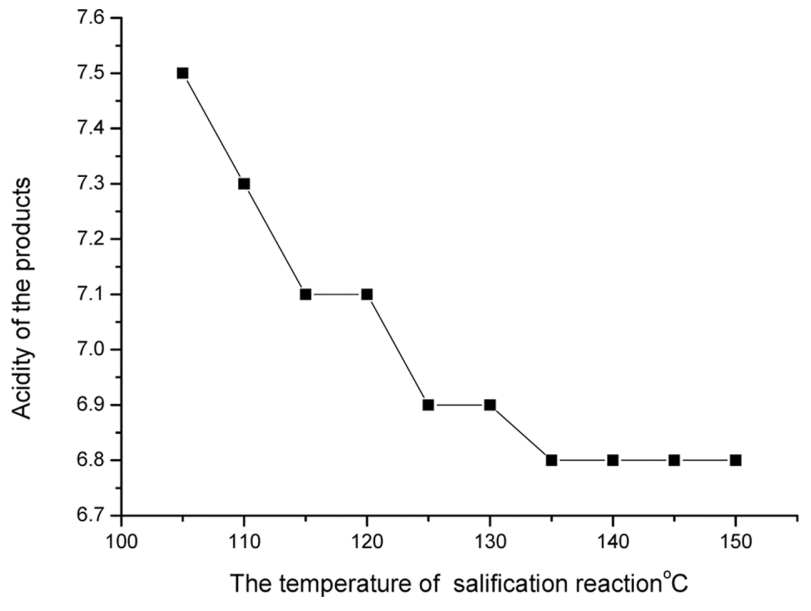

Fig. 4. The effect of temperature on the acidity of the salification reaction. Relationship between the temperature and acidity, the temperature from $100{ }^{\circ} \mathrm{C}$ to $150{ }^{\circ} \mathrm{C}$. The acidity of the sailfication reaction is decreased depending on the reaction temperature when the temperature above $135^{\circ} \mathrm{C}$ the acdity of the product is little changed. The temperature of salification reaction is $135^{\circ} \mathrm{C}$.

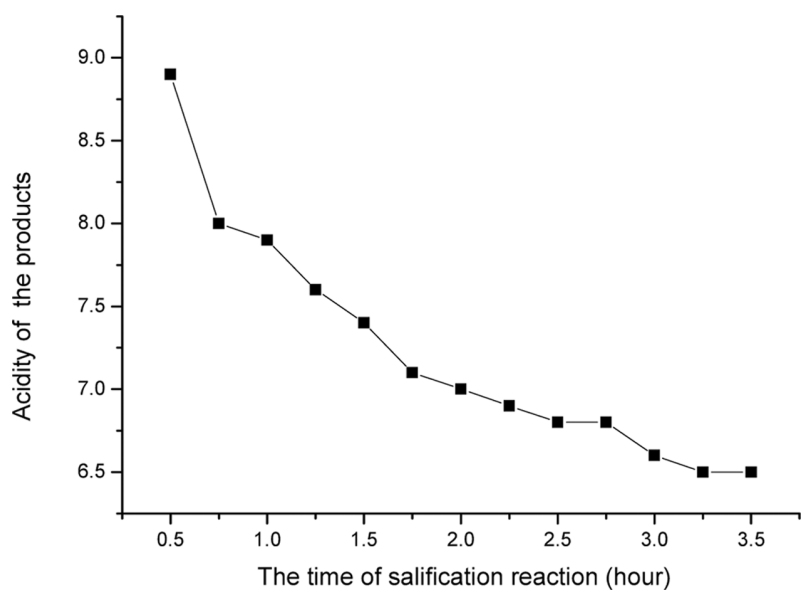

Fig. 5. The effect of time on the acidity of the salification reaction. Relationship between the time of salification reaction and acidity, reaction time from 0.5 end 3.5 hours. The acidity of the sailfication reaction is decreased depending on the reaction time. The appropriate acidity of product is between 6.5 and 7.5 . So choose the time of salification reaction at 3 hours. 


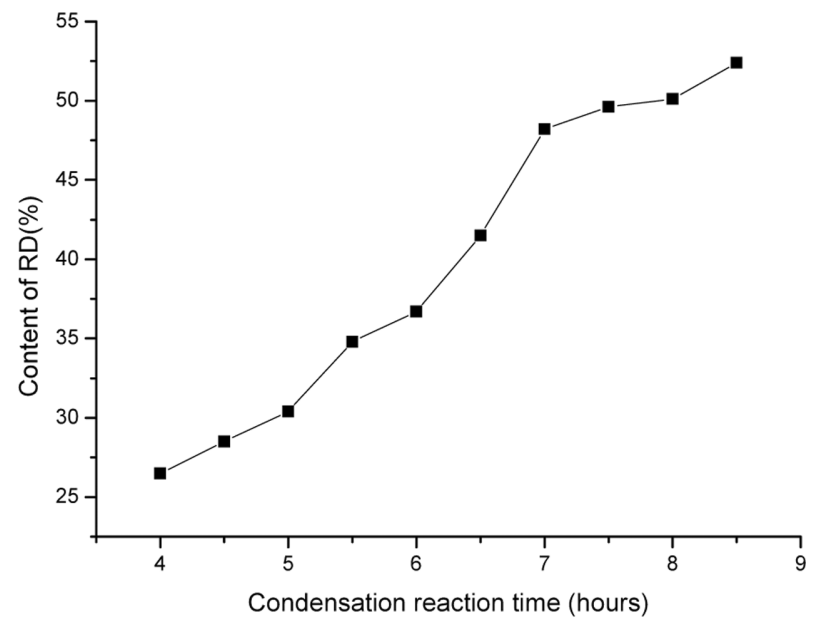

Fig. 6. The effect of condensation reaction time on content of $\mathrm{RD}$. Relationship between the condensation reaction time and content of RD, the reaction time from 4 end 9 hours. The content of $\mathrm{RD}$ is increased depending on the condensation reaction time. The content of RD should controlled above $45 \%$, so choose the time of condensation reaction at 7.5 hours.

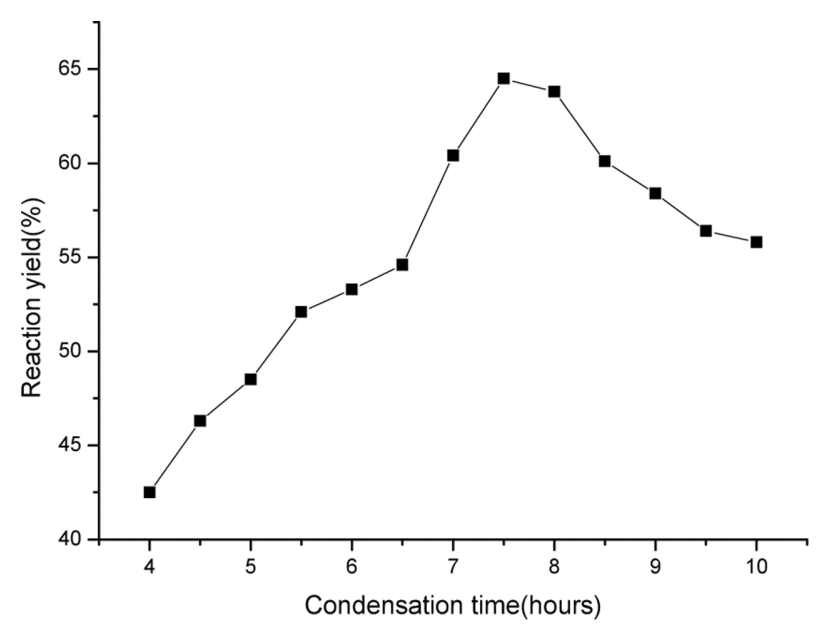

Fig. 7. The effect of condensation reaction time on reaction yield Relationship between the condensation reaction time and reaction yield of RD. The yield of RD is first increased depending on the condensation reaction time from 4 to 7.5 hours, then decreased after 7.5 hours. So choose the time of condensation reaction at 7.5 hours.

In the experiment we research acidity of aniline hydrochloride at different temperatures. Acidity of aniline hydrochloride controlled 6 to 8 , temperature and reaction time of condensation reaction is $135^{\circ} \mathrm{C}$ and 7.5 hours. Shown in Fig. 4-6 and Fig. 7.

It should be noted that this study has only examined factors and levels of the ratio of keto-amine, reaction time, reaction temperature and the ratio catalyst of acid-amine. The results do not imply the methods of charging order, heat transfer, acetone addition method and velocity and so on. Meanwhile adding monomer(or front fraction) is very importantly in the process of polymerization.

\section{REFERENCES}

1. Cyril, A.; Migdal, R. D. Abbott, U.S. Patent 6,726,855 B1, 2004.

2. Takashi Kojima, Ryozo Ishimoto, U.S. Patent 4,326,062, 1982.

3. Monsanto Chemical Company. Method of making improved polymeric di-hydroquinoline compositions U.S. Patent 3047521, July 31, 1962.

4. Goodrich, B. F. Company. Improvements in or relating to aniline condensation products and process of preparing same. Br. Patent 620314, May 23, 1949.

5. Goodrich, B. F. Company. Preparation of monomeric 2,2,4trimethyl-1,2-di-hydroquinoline. U.S. Patent 2514648, June $11,1950$.

6. Institute Organicheskoi Khimii lmeni N. D. Zelinskogo An SSSR. Process for the production of substituted 1,2dihydroquinolines. Br. Patent 1256725, December 15, 1971.

7. Acheson, R. M. An introduction to the chemistry of heterocyclic compounds, 1st ed.; Interscience Publishers inc.: London, 1962, p 223.

8. Heliodoro Monroy Rivera. Novel apparatus for the obtention of substituted 1,2-dihydroquinolines. U.S. Patent 3829292, August 13, 1974.

9. Heliodoro Monroy Rivera. Novel apparatus for the obtention of substituted 1,2-dihydroquinolines. U.S. Patent 3907507, September 23, 1975.

10. Elliott, I. W.; Dunathan, H. C. The structure of the dimer of 2,2,4-trimethy1-1,2-dihydroquinoline. Tetrahedron 1963, 19,833 .

11. Goodrich, B. F. Antioxidant. U.S. Patent 2048822, July 28, 1936.

12. Bridgestone Tire Company Limited. Antioxidant for Rubber. Br. Patent 2002783A. July 2, 1978.

13. Lattimer, R. P.; Hooser, E. R.; Zakriski, P. M. Characterization of aniline-Acetone condensation products by liquid chromatography and mass spectroscopy. Rubber Chem. Technol. 1980, 53(2), 346. 